\title{
Research on the first passage of double-layer micro-plate in the piezoelectric model
}

\author{
Zhenzi Zhang, Ping Wang* \\ College of Architectural Engineering and Mechanics, Yanshan University, Qinhuangdao 066000, China
}

Corresponding Author Email: Wangping0721@163.com

https://doi.org/10.18280/mmep.060115

Received: 7 January 2019

Accepted: 2 March 2019

\section{Keywords:}

MEMS, first passage, strain gradient

theory, stochastic average method

\begin{abstract}
Considering the macroscopic kinetic analysis model is not applicable to the MEMS field, this paper establishes an effective method for analyzing the stochastic stability of microcomponents. Firstly, it establishes a dynamic governing equation for the double-layer microplate simply supported on four sides based on the strain gradient theory and Hamilton's variational principle; then, it analyzes the first passage failure of the double-layer microplate in the piezoelectric model by using the stochastic average theory, and obtains the characteristic quantity of the first passage; finally, it compares the impacts of electrostatic excitation intensity on the first passage failure through numerical simulation. According to the results of the example analysis, even an excitation change that has a minimal impact on the overall failure process will significantly increase the probability of first passage failure in the rapid reliability degradation stage. The results obtained in this research can be used to extend the useful life of MEMS.
\end{abstract}

\section{INTRODUCTION}

The dynamics of micro-machinery is a science that studies the dynamic behaviors of mechanical systems in the medium and microscopic fields. With the research scope covering modeling and simulation of MEMS components, dynamic analysis and design, dynamics control, operational condition monitoring and fault diagnosis, it has very important theoretical significance and practical value.

On the microscopic scale, a material will show very different physical properties from those on the macroscopic scale. Such phenomenon, which is common in microstructures, is referred to as scale effect. In order to overcome this problem (which traditional mechanics cannot solve), Mandlin [1] proposed the classical couple stress theory in 1963, in which, the classical strain is symmetric while the couple stress is asymmetrical; in 2002, Yang et al. [2] proposed an isotropic modified couple stress theory, which makes both strain and curvature symmetric; in 2009, Tsiatas [3] established the Kirchhoff micro-plate model based on this theory; and in 2014, Shaat et al. [4] analyzed the bending problem of the Kirchhoff nano-plates with surface effects. At this point, the micro mechanical model for isotropic materials based on the modified couple stress theory is basically completed. However, in actual practice, micro-materials often have two or more layers, and the interlayer anisotropic impacts cannot be dealt with by the modified couple stress method. To solve this problem, both Chinese and foreign scholars have done a lot of work in the development of new theories in recent years. In 2010, Reddy et al. [5] proposed a nonlinear third-order theory for analyzing functionally graded material plates; In 2011, Chen et al. [6] proposed a stress gradient theory for anisotropic materials for the purpose of establishing a composite laminated beam model; in 2014, Li Anqing [7] figured out the number of independent high-order material constants in the isotropic strain gradient elasticity theory, which solved the basic theoretical problem in the constitutive relation. Reliability analysis is an indispensable part of material structure performance research. Now the theoretical basis for analyzing the reliability of stochastic dynamic systems from the perspective of first passage has been quite mature. In 2003, Zhu [8] established a complete process for studying the first passage failure problem; on this basis, in 2006, Li et al. [9] gave a method for solving the strong nonlinearity problem; in 2010, Zhu et al. [10] put forward a method for transforming a stochastic dynamic system into a quasi-integrable Hamiltonian system; in 2016, Ding et al. [11] analyzed the pull-in effect of electrostatically excited microbeams and gave a solution to the work of the electrostatic force; in 2017, L. Homsi et al. [12] proposed a method for deriving the Duffing equation based on the Galerkin method.

For basic structures like micro-plates and beam structures, the previous research mainly focused on the determination of static deformation and boundary conditions and the acquisition of vibration characteristics, etc., but rarely involves the life estimation and reliability analysis of these micro-structures in practice. This paper attempts to establish an effective method for assessing the reliability of MEMS components. By using the strain gradient theory and the stochastic averaging theory, it gives the first passage failure trend of the double-layer micro-plate, and then it compares the impacts of the electrostatic excitation with different intensities on the first passage of the double-layer micro-plate and specifies in which interval it is the most suitable to take measures to improve the performance of the double-layer micro-plate. This is a supplement to the reliability research of MEMS. 


\section{KINETIC MODEL FOR THE DOUBLE-LAYER MICRO-PLATE}

(1) Figure 1 shows an isotropic linear elastic double-layer micro-plate simply supported on four sides, with the upper and lower layers coinciding with each other. The plate has a length of a and a width of b. The thickness of the upper layer is $h_{l}$, and that of the lower layer is $h_{2}$. The plate is under a uniform load of $q(x, y, t)$ in the vertical direction. Suppose that the neutral plane is at a distance of $d$ from the contact surface. The displacement field is given based on the basic assumptions of the Kirchhoff plate:

(2)

$$
\left\{\begin{array}{l}
u=u_{0}(x, y, t)-z \frac{\partial w(x, y, t)}{\partial x} \\
v=v_{0}(x, y, t)-z \frac{\partial w(x, y, t)}{\partial y} \\
w=w(x, y, t)
\end{array}\right.
$$

where,

$$
u_{0}(x, y, t)=-d \frac{\partial w(x, y, t)}{\partial x}
$$

and

$v_{0}(x, y, t)=-d \frac{\partial w(x, y, t)}{\partial y}$

are the axial displacements on the contact surface.

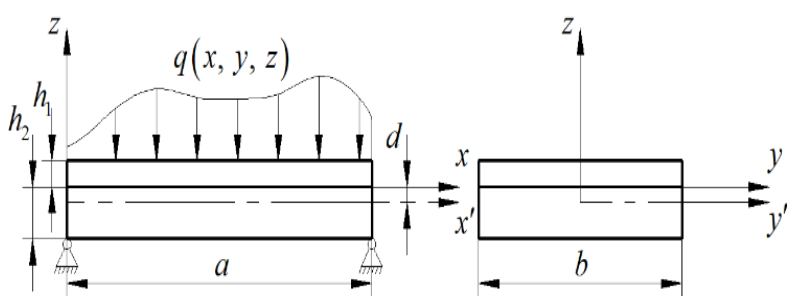

Figure 1. Geometric diagram of the double-layer micro-plate simply supported on four sides

According to the Cosserat theory with three high-order material parameters established based on the strain gradient theory in [7], for an isotropic linear elastic material, the strain energy density function of the modified couple stress is:

$$
\begin{aligned}
L= & \frac{1}{2} \lambda \varepsilon_{i i} \varepsilon_{j j}+\mu \varepsilon_{i j}^{\prime} \varepsilon_{i j}^{\prime}+\mu l_{0}^{2} \gamma_{i} \gamma_{i}+\mu l_{1}^{2} \eta_{i j k}^{(1)} \eta_{i j k}^{(1)}+ \\
& \mu\left(l_{2}^{2}+\frac{9}{5} l_{0}^{2}\right) \chi_{i j}^{\prime} \chi_{i j}^{\prime}+\mu\left(l_{2}^{2}+\frac{9}{5} l_{0}^{2}\right) \chi_{i j}^{\prime} \chi_{j i}^{\prime}
\end{aligned}
$$

where, $l_{0}, l_{1}$, and $l_{2}$ are the scale parameters of the material; and $\lambda$ and $\mu$ are the Lame coefficients.

Substitute non-zero strain and strain gradient tensor into the above equation to obtain the strain energy density of a single layer. Then calculate the integral sum of the strain energy functions of the two layers to obtain the strain energy of the double-layer micro-plate.

$$
U=\iint_{A}\left(\int_{-h_{1}}^{0} L_{1} \mathrm{~d} z\right) \mathrm{d} S+\iint_{A}\left(\int_{0}^{h_{2}} L_{2} \mathrm{~d} z\right) \mathrm{d} S
$$

where, $L_{1}$ and $L_{2}$ are respectively the strain energy density functions of the upper and the lower plates.

In the electrostatic actuated model shown in Figure 2, the upper polar plate is a double-layer micro-plate simply supported on four sides, subjected to an electrostatic force of $q(x, y, t)$ in the vertical direction, and the lower plate is fixed. The distance between the two plates is $g$.

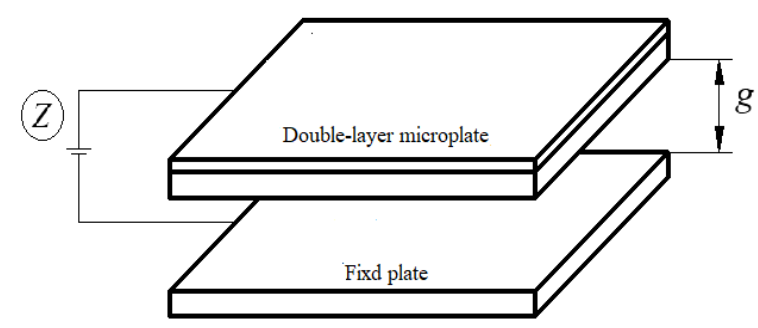

Figure 2. Electrostatic actuated model

The kinetic energy of the double-layer micro-plate is:

$T=\frac{1}{2} \iiint_{v}\left(\rho_{1}+\rho_{2}\right) \dot{w}^{2} \mathrm{~d} V$

where, $\mathrm{w}=\frac{\partial w}{\partial t}$, and $\rho_{1}$ and $\rho_{2}$ are the density of the upper and lower plates, respectively.

The work of the electrostatic force is:

$W=\iint_{A} q(x, y, t) w(x, y, t) \mathrm{d} S$

The electrostatic force $\mathrm{q}$ consists of the electrostatic actuation force $\mathrm{q}_{\mathrm{e}}$, the film damping force $\mathrm{q}_{\mathrm{c}}$ and the noise term $\mathrm{q}^{\prime} \delta(t)$

$q=q_{e}+q_{c}+q^{\prime} \xi(t)$

When the upper and lower polar plates completely coincide with each other [14]:

$q_{e}=\frac{\varepsilon_{0} b Z^{2}(t)}{2(g-w)^{2}}$ and $q_{c}=-\frac{c_{s} b^{3}}{(\mathrm{~g}-w)^{3}} \dot{w}$

In the case of AC loading [11]:

$$
\begin{aligned}
Z^{2}(t) & =\left[v_{a c} \cos \left(\left(w^{\prime} t\right)\right)\right]^{2} \\
& =\frac{1}{2} v_{a c}^{2}\left[1+\cos \left(2 w^{\prime} t\right)\right]
\end{aligned}
$$

where, q' is the noise amplitude; $\delta(t)$ the Gaussian white noise with a mean of 0 and an intensity of $2 D ; \varepsilon_{0}$ the permittivity of vacuum, $\varepsilon_{s}$ the air viscosity coefficient, $Z(t)$ the on-load voltage, $v_{a c}$ the on-load voltage amplitude, and $w^{\prime}$ the frequency of the on-load voltage. 
Apply Hamilton's principle:

$\int_{t_{1}}^{t_{2}}[\delta T-(\delta U-\delta W)] \mathrm{d} t=0$

Substitute equations 3-5 into the above equation and observe the transverse vibration in the vertical direction to obtain the governing equation for the lateral vibration of the double-layer micro-plate:

$$
\begin{aligned}
& -2 c_{1} \nabla^{6} w+2 c_{2} \nabla^{4} w+2 c_{3}\left(\frac{\partial^{5} u_{0}}{\partial x^{5}}+2 \frac{\partial^{5} u_{0}}{\partial x^{3} \partial y^{2}}+\right. \\
& \left.\frac{\partial^{5} u_{0}}{\partial x \partial y^{4}}+\frac{\partial^{5} u_{0}}{\partial x^{4} \partial y}+2 \frac{\partial^{5} u_{0}}{\partial x^{2} \partial y^{3}} \frac{\partial^{5} u_{0}}{\partial y^{5}}\right)-2 c_{4}\left(\frac{\partial^{3} u_{0}}{\partial x^{3}}+\right. \\
& \left.\frac{\partial^{3} u_{0}}{\partial x \partial y^{2}}+\frac{\partial^{3} v_{0}}{\partial x^{2} \partial y}+\frac{\partial^{3} v_{0}}{\partial y^{3}}\right)+\left(\rho_{1} h_{1}-\rho_{2} h_{2}\right) \ddot{w}+q=0
\end{aligned}
$$

where,

$$
\begin{aligned}
c_{1}= & h_{1}^{3}\left(\frac{3}{5} \mu_{1} l_{0(1)}^{2}+\frac{2}{15} \mu_{1} l_{2(1)}^{2}\right)+\frac{h_{2}^{3}}{3}\left(\frac{3}{5} \mu_{2} l_{0(2)}^{2}+\frac{2}{15} \mu_{2} l_{2(2)}^{2}\right) \\
c_{2}= & h_{1}\left(\frac{6}{5} \mu_{1} l_{0(1)}^{2}+\frac{4}{15} \mu_{1} l_{1(1)}^{2}+\mu_{1} l_{2(1)}^{2}\right)+\frac{E_{1} h_{1}^{3}}{6\left(1-\lambda_{1}^{2}\right)}+ \\
& h_{2}\left(\frac{6}{5} \mu_{2} l_{0(2)}^{2}+\frac{4}{15} \mu_{2} l_{1(2)}^{2}+\mu_{2} l_{2(2)}^{2}\right)+\frac{E_{2} h_{2}^{3}}{6\left(1-\lambda_{2}^{2}\right)} \\
c_{3}= & -h_{1}^{2}\left(\frac{9}{10} \mu_{1} l_{0(1)}^{2}+\frac{1}{5} \mu_{1} l_{2(1)}^{2}\right)+h_{2}^{2}\left(\frac{9}{10} \mu_{2} l_{0(2)}^{2}+\frac{1}{5} \mu_{2} l_{2(2)}^{2}\right) \\
c_{4}= & -\frac{E_{1} h_{1}^{2}}{4\left(1-\lambda_{1}^{2}\right)}+\frac{E_{2} h_{2}^{2}}{4\left(1-\lambda_{2}^{2}\right)}, \nabla^{4}=\frac{\partial^{4}}{\partial x^{4}}+2 \frac{\partial^{4}}{\partial x^{2} \partial y^{2}}+\frac{\partial^{4}}{\partial y^{4}} \\
\nabla^{6}= & \frac{\partial^{6}}{\partial x^{6}}+3 \frac{\partial^{6}}{\partial x^{4} \partial y^{2}}+3 \frac{\partial^{6}}{\partial x^{2} \partial y^{4}}+\frac{\partial^{6}}{\partial y^{6}},
\end{aligned}
$$

$E_{1}, \mu_{1}, \lambda_{1}, l_{0(1)}, l_{1(1)}$ and $l_{2(1)}$ are the material parameters of the upper plate, and $E_{2}, \mu_{2}, \lambda_{2}, l_{0(2)}, l_{1(2)}$ and $l_{2(2)}$ are those of the lower plate.

The corresponding boundary conditions are:

$w(0, y, t)=w(a, y, t)=0, w(x, 0, t)=w(x, b, t)=0$,

$v_{0}(0, y, t)=v_{0}(a, y, t)=0, u_{0}(x, 0, t)=u_{0}(x, b, t)=0$,

$v_{0 x}(0, y, t)=v_{0 x}(a, y, t)=0, u_{0 y}(x, 0, t)=u_{0 y}(x, b, t)=0$,

$w_{x}(0, y, t)=w_{x}(a, y, t)=0, w_{y}(x, 0, t)=w_{y}(x, b, t)=0$

where,

$w_{x}=\frac{\partial w}{\partial x}, w_{y}=\frac{\partial w}{\partial y}, v_{0 x}=\frac{\partial v_{0}}{\partial x}$

and

$$
u_{0 y}=\frac{\partial u_{0}}{\partial y} .
$$

\section{DERIVATION OF \\ THE \\ HAMILTON \\ DIFFERENTIAL EQUATION}

Considering the boundary conditions of the double-layer micro-plate simply supported on four sides, the flexural trial function that satisfies the boundary conditions is selected, as below:

$$
w(x, y, t)=K(t) \sin \frac{\pi}{a} x \sin \frac{\pi}{b} y
$$

Substitute the above equation into governing equation (2$10)$ and apply the Galerkin integral:

$$
\begin{aligned}
& \iint_{A}\left[M K \sin \frac{\pi}{a} x \sin \frac{\pi}{b} y+N K \cos \frac{\pi}{a} x \cos \frac{\pi}{b} y+\right. \\
& \left(\rho_{1} \mathrm{~h}_{1}-\rho_{2} \mathrm{~h}_{2}\right) \ddot{K} \sin \frac{\pi}{a} x \sin \frac{\pi}{b} y-\dot{K} \frac{c_{s} b^{3}}{g} \sin \frac{\pi}{a} x \sin \frac{\pi}{b} y- \\
& \left.\frac{\varepsilon_{0} b Z^{2}}{4 g^{3}}\left(\mathrm{~g}+2 K \sin \frac{\pi}{a} x \sin \frac{\pi}{b}\right)+q^{\prime} \xi(t)\right] \sin \frac{\pi}{a} x \sin \frac{\pi}{b} y \mathrm{~d} S=0
\end{aligned}
$$

Simplify the above equation to obtain the Duffing equation for the double-layer micro-plate:

$$
\ddot{K}+\alpha \dot{K}+\beta K+\gamma K+\lambda+\chi \xi(t)=0
$$

where,

$$
\begin{aligned}
\alpha= & -\frac{b^{3} c_{s}}{g^{3}\left(\rho_{1} h_{1}-\rho_{2} h_{2}\right)}, \beta=\frac{M}{\rho_{1} h_{1}-\rho_{2} h_{2}} \\
\gamma= & \frac{Z^{2} b \varepsilon_{0}}{2 g^{3}\left(\rho_{1} h_{1}-\rho_{2} h_{2}\right)}, \lambda=\frac{4 Z^{2} b \varepsilon_{0}}{\pi^{2} g^{2}\left(\rho_{1} h_{1}-\rho_{2} h_{2}\right)} \\
\chi= & \frac{16 q^{\prime}}{\pi^{2}\left(\rho_{1} h_{1}-\rho_{2} h_{2}\right)}, \ddot{K}=\frac{\partial^{2} K}{\partial t^{2}}, \dot{K}=\frac{\partial K}{\partial t} \\
N= & -d\left[2 c_{3}\left(\frac{\pi}{a}\right)^{5} \frac{\pi}{b}+4 c_{3}\left(\frac{\pi}{a}\right)^{3}\left(\frac{\pi}{b}\right)^{3}+2 c_{3} \frac{\pi}{a}\left(\frac{\pi}{b}\right)^{5}-\right. \\
& \left.2 c_{4}\left(\frac{\pi}{a}\right)^{3} \frac{\pi}{b}-2 c_{4} \frac{\pi}{a}\left(\frac{\pi}{b}\right)^{3}\right]
\end{aligned}
$$

And

$$
\begin{aligned}
M= & 2 c_{1}\left(\frac{\pi}{a}\right)^{6}+2 c_{1}\left(\frac{\pi}{b}\right)^{6}+6 c_{1}\left(\frac{\pi}{a}\right)^{4}\left(\frac{\pi}{b}\right)^{2}+ \\
& 6 c_{1}\left(\frac{\pi}{a}\right)^{2}\left(\frac{\pi}{b}\right)^{4}+2 c_{2}\left(\frac{\pi}{a}\right)^{4}+4 c_{2}\left(\frac{\pi}{a}\right)^{2}\left(\frac{\pi}{b}\right)^{2}+ \\
& 2 c_{2}\left(\frac{\pi}{b}\right)^{4}-d\left[2 c_{3}\left(\frac{\pi}{a}\right)^{6}+4 c_{3}\left(\frac{\pi}{a}\right)^{4}\left(\frac{\pi}{b}\right)^{2}+\right. \\
& \left.2 c_{3}\left(\frac{\pi}{a}\right)^{2}\left(\frac{\pi}{b}\right)^{4}-2 c_{4}\left(\frac{\pi}{a}\right)^{4}-4 c_{4}\left(\frac{\pi}{a}\right)^{2}\left(\frac{\pi}{b}\right)^{2}\right]
\end{aligned}
$$




\section{CALCULATION OF THE CHARACTERISTIC QUANTITY OF THE FIRST PASSAGE}

Let $q=K$ and $p=K$, and the Hamiltonian equations for the movement of the governing system are obtained:

$\left\{\begin{array}{l}\dot{q}=p \\ \dot{p}=-\alpha p-\beta q-\gamma q-\lambda-\chi \xi(t)\end{array}\right.$

Considering the quasi non-integrable Hamiltonian system with dissipation under the actuation of the electrostatic force, from the stochastic averaging principle of the quasi nonintegrable Hamiltonian system, it can be seen that the vibration system converges to the one-dimensional diffusion process, and its drift and diffusion coefficients are:

$$
\left\{\begin{array}{l}
\bar{m}(H)=\frac{1}{T(H)} \int_{\Omega}\left[-\alpha p+\frac{\chi^{2}}{2 p}\right] \mathrm{d} q \\
\bar{\sigma}^{2}(H)=\frac{1}{T(H)} \int_{\Omega} \chi^{2} p \mathrm{~d} q \\
T(H)=\int_{\Omega} \frac{1}{p} \mathrm{~d} q
\end{array}\right.
$$

The integral domain is:

$\Omega=\{(q, 0) \mid H(q, 0) \leq H\}$.

Take the Hamiltonian function from equation (13) as follows:

$H=\frac{1}{2}\left(p^{2}+\beta q^{2}+\gamma q^{2}\right)+\lambda q$

Let $p=-R \sin x$ and $q=R \cos x$, and substitute them into the above equation to simplify the Hamiltonian equation as follows:

$H_{0}=\frac{R^{2}(\beta+\gamma)}{2}+\lambda R$

Substitute the positive root

$R=\frac{-\lambda+\sqrt{\lambda^{2}+2 H_{0}(\beta+\gamma)}}{\beta+\gamma}$

and $\mathrm{p}$ and $\mathrm{q}$ back into equation (15) to obtain the drift and diffusion coefficients:

$$
\left\{\begin{array}{l}
\bar{m}\left(H_{0}\right)=-\frac{\alpha\left[-\lambda+\sqrt{\lambda^{2}+2 H_{0}(\beta+\gamma)}\right]^{2}}{2(\beta+\gamma)^{2}}+\frac{\chi^{2}}{2} \\
\bar{\sigma}^{2}\left(H_{0}\right)=\frac{\chi^{2}\left[-\lambda+\sqrt{\lambda^{2}+2 H_{0}(\beta+\gamma)}\right]^{2}}{2(\beta+\gamma)^{2}} \\
T\left(H_{0}\right)=\pi
\end{array}\right.
$$

According to the conclusion given in [15], when the Hamilton function tends to be $0, H_{0}=0$ is the first kind of singularity and is a flow point. The corresponding drift and diffusion indices and characteristic values are:

$\beta_{s}=1, \alpha_{s}=1$ and $c_{l}=\frac{2 \alpha}{\chi^{2}}$

$H_{0}=0$ means entering the boundary. The trivial solution to the system does not have a stable probability, and thus there is the first passage problem.

\section{EXAMPLE ANALYSIS}

Take the physical parameters of the double-layer microplate under the actuation by the electrostatic force as follows: $\mathrm{a}=30 \mu \mathrm{m}, \mathrm{b}=20 \mu \mathrm{m}, h_{1}=0.8 \mu \mathrm{m}, h_{2}=1.6 \mu \mathrm{m}, g=12 \mu \mathrm{m}$, $E_{1}=130 \mathrm{GPa}, E_{2}=85 \mathrm{GPa}, l_{1(1)}=l_{2(1)}=l_{3(1)}=5.9 \mu \mathrm{m}$, $l_{1(2)}=l_{2(2)}=l_{3(2)}=7.8 \mu \mathrm{m}, \lambda_{1}=\lambda_{2}=0.3, \mu_{1}=\mu_{2}=0.5$, $\mathrm{H}_{0}=0.001, q^{\prime}=1, \rho_{1}=\rho_{2}=2.6 \times 10^{3} \mathrm{~kg} / \mathrm{m}^{3}, \varepsilon_{0}=8.85 \times$ $10^{-12}$ and $\varepsilon_{s}=2 \times 10^{-5}$.

Solve the generalized Kolmogorov equation to obtain the $k$ -th moment of the first passage time. Let $k=1$, and we have Pontryagin's equation describing the average first passage time, which is of the greatest interest in practice:

$\frac{1}{2} \bar{\sigma}^{2}\left(H_{0}\right) \frac{\mathrm{d}^{2} \mu_{k}\left(H_{0}\right)}{\mathrm{d} H_{0}^{2}}+\bar{m}\left(H_{0}\right) \frac{\mathrm{d} \mu_{k}\left(H_{0}\right)}{\mathrm{d} H_{0}}=-1$

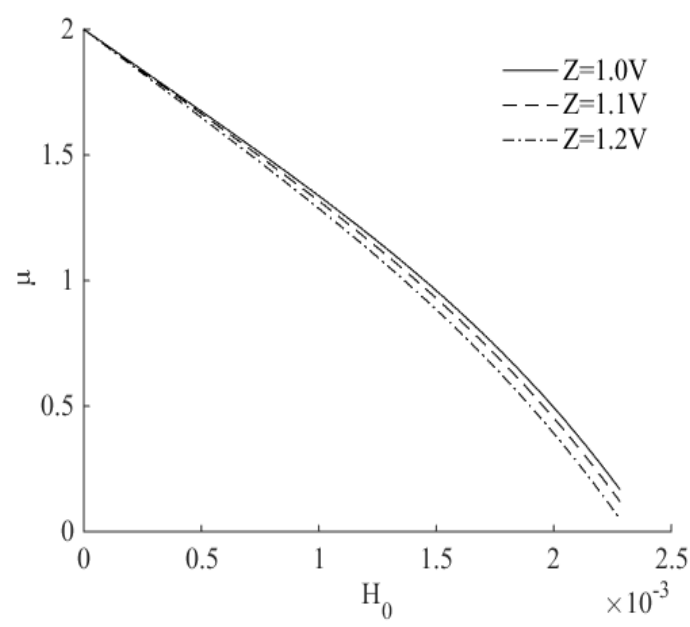

Figure 3. Average first passage time under electrostatic excitation with different intensities

Figure 3 shows the relationship between the first passage time $\mu_{1}\left(H_{0}\right)$ and the total energy $H_{0}$ of the system under different intensities of electrostatic excitation. As the total energy of the system increases, the first passage takes less and less time on average and will fail before the total energy reaches the right boundary. When the excitation intensifies, the total energy accumulation rate of the system will increase and will pass the safety domain earlier. In order to more clearly describe the first passage phenomenon, the drift and diffusion coefficients are substituted into the Kolmogorov backward equation to obtain the conditional reliability function $\mathrm{R}(\mathrm{t})$ : 
$\frac{\partial R}{\partial t}=\bar{m}\left(H_{0}\right) \frac{\partial R}{\partial H_{0}}+\frac{1}{2} \bar{\sigma}^{2}\left(H_{0}\right) \frac{\partial^{2} R}{\partial H_{0}^{2}}$

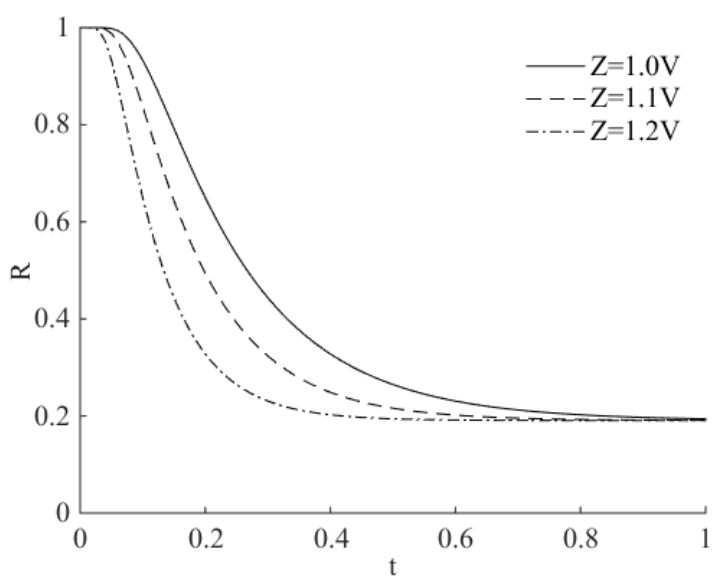

Figure 4. Conditional reliability function under electrostatic excitation with different intensities

For a particular system, the time corresponding to the allowed range of the conditional reliability function is the design life. It can be seen from the simulation results in Fig. 4 that the reliability function decreases slowly within a certain period after the system is just put into use, and the system is the most stable at this time; as the service time increases, the degradation speed increases gradually; when the failure region is approached, the degradation speed slows down again, which is in line with the practical application experience. When the input excitation increases, the image degradation rate increases and the system fails earlier. Although the overall gap is still small, it already shows the impact of small excitation changes on the local reliability of the system. The conditional probability density function of the first passage time $\tau$ is further introduced:

$p\left(\tau \mid H_{0}\right)=\left.\frac{\partial P_{f}\left(t \mid H_{0}\right)}{\partial t}\right|_{t=\tau}=-\left.\frac{\partial R\left(t \mid H_{0}\right)}{\partial t}\right|_{t=\tau}$

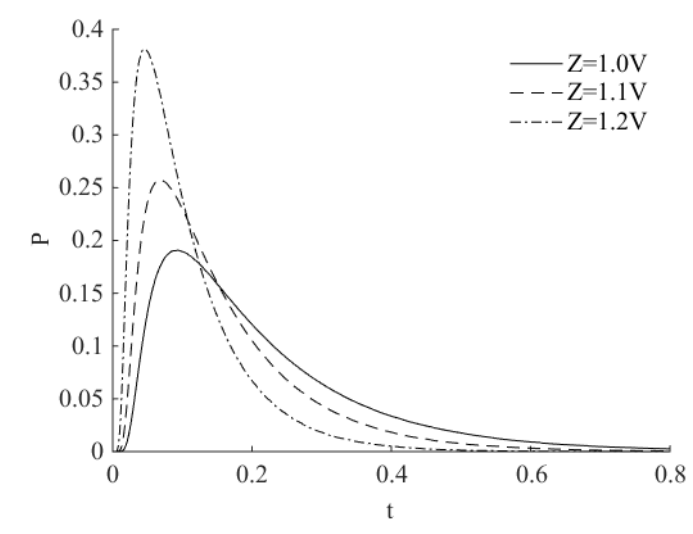

Figure 5. Conditional probability density of the first passage time under electrostatic excitation with different intensities

In the physical sense, the conditional probability density of the first passage time refers to the conditional probability density of the system damage at a certain point in time. It can be seen from the simulation results in Figure 5 that larger excitation corresponds to a higher and earlier peak. The vibration state of the system near the peak is the most complex, and the probability of first passage failure is the highest. It is worth noting that the peak value corresponding to $Z=1.2 \mathrm{~V}$ is close to twice that when, indicating that when the system reliability is rapidly reduced, even a small excitation change to the overall failure process will bring about a very significant local difference.

\section{CONCLUSIONS}

This paper compares the average first passage time $\mu_{1}\left(H_{0}\right)$, the conditional reliability function $\mathrm{R}(\mathrm{t})$ and the conditional probability density of the first passage time $p\left(\tau \mid H_{0}\right)$ under excitation with different intensities, respectively, and obtains the following conclusions:

(1) As the service time of the piezoelectric system increases, the degradation rate of the structural reliability is slow first, and then increases and at last slows down again.

(2) Although a small change in the excitation intensity has no obvious effect on the overall failure process, when the system enters the stage of rapid reliability degradation, a minimal excitation change can bring a huge difference in peak values.

(3) For a MEMS system, the vicinity of the peak $p\left(\tau \mid H_{0}\right)$ is a dangerous interval where first passage failure is likely to occur, and also the best range for eliminating vibration deformation, achieving precise positioning control and improving the smooth operation of the system.

This paper establishes a method for analyzing the first passage failures of micro-components. The research results obtained can be used as reference in the reliability analysis of piezoelectric thin-plate structures in engineering practice.

\section{ACKNOWLEDGMENT}

Hebei Natural Science Foundation: A2016203101.

\section{REFERENCES}

[1] Mindlin RD. (1963). Influence of couple stresses on stress concentrations. Experimental Mechanics 3(1): 1-7. https://doi.org/10.1016/0043-1648(63)90084-X

[2] Yang F, Chong AM, Lam DC, Tong P. (2002). Couple stress based on strain gradient theory for elasticity. International Journal of Solids and Structures 39N(10): 2731-2743. https://doi.org/10.1016/s00207683(02)00152-x

[3] Tsiatas GC. (2009). A new Kirchhoff plate model based on a modified couple stress theory. International Journal of Solids and Structures 46(13): 2757-2764. https://doi.org/10.1016/j.ijsolstr.2009.03.004

[4] Shaat M, Mahmoud FF, Gao XL, Faheem Ahmed F. (2014). A Size dependent bending analysis of Kirchhoff plates based on a modified couple stress theory including surface effects. International Journal of Machanical Sciences 79:

31-37. https://doi.org/10.1016/j.ijmecsci.2013.11.022

[5] Reddy JN, Kim J. (2012). A nonlinear modified couple stress based on third order theory of functionally graded plates. Composite Structures 94(3): 1128-1143. 
https://doi.org/10.1016/j.compstruct.2011.10.006

[6] Chen WJ, Li L, Ma X. (2011). A modified couple stress model for bending analysis of composite laminated beams with first order shear deformation. Composite Structures $\quad 93(11)$ : 2723-2732. https://doi.org/10.1016/j.compstruct.2011.05.032

[7] Li AQ, Zhou SJ. (2014). A size-dependent bilayered microbeam model based on strain gradient elasticity theory. Composite Structures 108(1): 259-266. https://doi.org/10.1016/j.compstruct.2013.09.020

[8] Zhu WQ, Wu YJ, Chen LC. (2003). First-passage time of duffing oscillator under combined harmonic and white-noise excitation. Nonlinear Dynamics 32(3): 291 305. https://doi.org/10.1016/j.probengmech.2018.06.003

[9] Li W, Xu W, Zhao JF, Jin YF. (2006). First-passage problem for strong nonlinear stochastic dynamical systems. Chaos Solitons \& Fractals 28(2): 414-421. https://doi.org/10.1016/j.chaos.2005.05.054

[10] Chen LC, Zhu WQ. (2010). First passage failure of quasi partial integrable generalized Hamiltonian systems. International Journal of Non-Linear Mechanics 45(1): 56-62. https://doi.org/10.1016/j.ijnonlinmec.2009.09.002

[11] Ding N, Xu X, Zheng ZQ, Li E. (2017). Size-dependent nonlinear dynamics of a microbeam based on the modified couple stress theory. Acta Mechanica 228(10): 3561-3579. https://doi.org/10.1007/s00707-017-1895-3

[12] Homsi L, Geuzaine C, Noels L. (2017). A coupled electro-thermal Discontinuous Galerkin method. Journal of Computational Physics 348: 231-258. https://doi.org/10.1016/j.jcp.2017.07.028

[13] Wu J, Cao J. (2013). Generic mechanism of optimal energy transfer efficiency: a scaling theory of the mean first-passage time in exciton systems. Physical Review Letter 110(20): 2624-2628. https://doi.org/10.1103/physrevlett.110.200402

[14] Zhang WM, Meng G. (2007). Nonlinear dynamic analysis of electrostatically actuated resonant mems sensors under parametric excitation. IEEE Sensors Journal 7(3-4): 370-380. https://doi.org/10.1109/jsen.2006.890158

[15] Zhu WQ, Ying ZG, Soong TT. (2001). An optimal nonlinear feedback control strategy for randomly excited structural systems. Nonlinear Dynamics 24(1): 31-35. https://doi.org/10.1023/a:1026527404183

[16] Mergen HG, Marco A, Hamed F. (2013). Nonlinear forced vibrations of a microbeam based on the strain gradient elasticity theory. International Journal of Engineering Science 63: 52-60. https://doi.org/10.1016/j.ijengsci.2012.12.001

[17] Kahrobaiyan MH, Asghari M, Ahmadian MT. (2014). A Timoshenko beam element based on the modified couple stress theory. International Journal of Mechanical Sciences 79: 75-83. https://doi.org/10.1016/j.ijmecsci.2013.11.014

[18] Tao C, Fu YM. (2017). Thermal buckling and post buckling analysis of size dependent composite laminated micro-beams based on a new modified couple stress theory. Acta Mechanica 228(5): 1711-1724. https://doi.org/10.1007/s00707-016-1770-7

[19] He D, Yang WL, Chen WJ. (2017). A size dependent composite laminated skew plate model based on a new modified couple stress theory. Science Direct 30(1): 7586. https://doi.org/10.1016/j.camss.2016.12.001 